\title{
APPLICATION OF CLOUD COMPUTING FOR PROVIDING KNOWLEDGE MANAGEMENT SERVICES FOR HIGHER EDUCATION SECTOR
}

\author{
Muhammad Rafiq a , Abul Bashar ${ }^{\mathrm{b}}$, Arifusalam Shaikh c, \\ abc Prince Mohammad Bin Fahd University KSA, Saudi Arabia \\ Corresponding email: abashar@pmu.edu.sa
}

\begin{abstract}
Higher education institutions in the private sector are under budgetary constraints when it comes to developing their IT infrastructure to provide varied services to the students, the faculty and the staff. This is in contrast to their public sector counterparts who are abundantly funded by the government. In order to optimize their spending and cut down on the capital expenditure and operation expenditure for setting up an IT infrastructure, the private universities resort to the Cloud Computing model of leasing infrastructure from cloud providers. This then enables them to run their in-house developed applications or outsource the application development and provisioning to cloud service providers. In this backdrop, the paper presents a case study of providing Knowledge Management Service (KMS) to the IT end users of the university which runs on the leased cloud infrastructure. The motivation for the choice of this service was that KMS effectively enhances the efficiency and facilitates the ongoing knowledge transfer for the progress of the university. To this end, a methodology is proposed to provide a platform to build a decision support system for private universities to help them in procuring Cloud Computing facility and to develop the KMS for the IT users. It also presents initial survey results of the potential of adaptability of Cloud Computing services by enlisting and measuring their merits and drawbacks.
\end{abstract}

Keywords: Cloud Computing Model, IT Infrastructure \& Knowledge Management Systems.

\section{Introduction}

Public Universities are generally well funded and enjoy heavy budgets for their sustained development programs and strategic initiatives. However, private universities are always constrained with limited budgets and expect higher returns for limited investments. KMS have proved to enhance efficiency and facilitate ongoing knowledge transfer process. Among other benefits of KMS is to ease knowledge sharing, automate processes, foster collaboration, increase productivity, and to make an integration with IT, but requires investments in such heavy infrastructure. Cloud computing (CC) services can thus be utilized to avail customized infrastructure to develop KMS progressively within the financial constraints. Knowledge Management is now considered as a crucial embedded component in any organization including educational institutes. Since the prime focus is knowledge in educational institutes, various methodologies are now being developed and implemented to reap the benefits.

The importance of having KM in higher education institutes (HEI) is thoroughly acknowledged but the challenge now is to analyze the requirements in terms of technology that can efficiently support such initiatives. Without any doubt it is clear that a sound IT platform with state of art infrastructure is desired for implementing KMS in any organization. However, this may require heavy investment if desired to build in-house. The emergence of cloud computing services has come to the rescue especially for small and medium scale organizations who can rent the 
services as needed. Similar solutions can now be sought by HEIs as well to provide services in cost effective manner.

Cloud computing is gaining popularity because of its variety of services offered like resource pooling, on-demand service, rapid elasticity, pay-as-you-go, network access, etc. Topped up with the added benefits like lower IT costs, unlimited storage, latest software updates, easier collaboration and device freedom it makes an unique choice for small and medium scale organization to exploit its benefits. With regard to higher education, most of the private colleges/universities generally fall under the SME category. Therefore, the potential of adapting to $\mathrm{CC}$ is high for general IT services. However, a careful analysis is necessary if the university is adopting $\mathrm{CC}$ to utilize it for developing a KMS.

While CC brings great advantages it is still prone to some risk elements that include the data security risk. Utilizing CC is always associated with a threat that the security and privacy of the organization's data and information would be compromised. However, this demerit has prompted extensive research work in the field of cloud security and its services. Hence this promise is to be taken positively and proceeded further in adapting CC services, including for developing KMS.

The proposed study analyzes the prospects of developing KMS for private higher education universities utilizing Cloud Computing services. A methodology in the form of a roadmap is developed that can be utilized to build a strategic Knowledge Management System by availing the Cloud Computing services for small and medium level Higher Education Institutes (HEI). The objective of this paper is to identify the basic requirements for an HEI to adopt a CC service and develop a methodology in building a KMS.

\section{Review of Literature}

The current research aims at providing a framework to develop knowledge management systems by utilizing cloud computing services for higher education institutes. There is an abundance of literature to know the benefits, limitations and applications of knowledge management systems. However, adaptation of $\mathrm{KM}$ in higher education has been relatively a new trend. Further adopting cloud computing services at higher education institutes is also a fairly new concept. Therefore, the following literature review will be focusing on recent papers that discuss about developing KMS for higher education and how cloud computing is making its space in the higher education.

Researchers have presented knowledge management system as a framework of an integration of organizational elements in organizational culture, organizational information technology infrastructure and the organization's store of individual and collective experiences, learning, insights, values (Allee, 1997). These characteristics are also valid for HEIs. The increasing demand in online offerings and other support services for students by HEIs can be considered the key reasons for the adoption of KM. Some of the benefits (Milam, 2001) that HEIs can gain through the adoption of KM include to

- increase student retention and graduation rates

- retain a technology workforce in the face of severe employee shortages

- expand new web based offerings

- work to analyze the cost effective use of technology to meet more enrolment 
- transform existing transaction-based systems to provide information, not just data, for management and

- compete in an environment where institutions cross state and national borders to meet student needs ubiquitously.

Coukos-Semmel, (2003) categorized knowledge as academic and organizational knowledge in the higher education environment. While academic knowledge is the primary purpose of the institutions, organizational knowledge deals with the overall business of the institute. According to Cranfield \& Taylor (2008) HEIs basically have two distinct cultures, an academic culture and an administrative culture which may further have sub-cultures within their discipline or functions. They concluded that enough cultural management is not exercised in practice. While universities do share knowledge with their students, and use committees to make decisions and share information and knowledge, there is a need to understand the additional benefits of adopting KM. Kok (2007) studied the importance of managing and measuring intellectual capital as part of their knowledge management initiatives. He presented a framework that can be used to manage and measure Intellectual capital at any institution of higher education. Brewer \& Brewer (2010) examined the relationship between KM, human resource management, and typical knowledge learning goals of an accredited business education program. It is known that KM enhances competitive advantage of any organization, therefore if better understood and utilized in higher education institutes, it can also aid in preparing better graduates and could be a good way to measure assurance of learning.

Rowley, (2000) addressed four main objectives of KM projects, which are to create knowledge repositories, to improve knowledge access, to enhance the knowledge environment and to manage knowledge as an asset. Further, she also identified several challenges to adopt an effective KM system such as change in culture and values, organizational structures and reward systems, managing relationship between knowledge and power etc. Universities may have a basic KMS but researchers have suggested to refine KM in education, to connect KM approach to organization's outcome and to distinguish KM approach from information system and technology implementation (Petrides, 2003).

Bimol, et al (2014) discuss the financial constraints and the world economy that are affecting higher education institutions so as to provide the necessary information technology support for knowledge management activities. They argue that Cloud computing offers a cost-effective and viable solution to such a scenario through its characteristic features of on-demand service provisioning and pay-as-you-go payment model. In their paper they discuss that Cloud model applications can obtain the advantage of working ability and communication in educational environment without taking into account the constraints of time and space. Hence, Cloud Computing provides a new solution to Knowledge management IT infrastructure problem by integrating in higher education by establishing a unified, open, simple and flexible architecture. Sharma, et al (2013) present a complex problem in current computing environment where the hardware and software are changing at a very rapid speed and the institutes of higher education suffer the burden of high cost of purchase of computing resources and later the hassle of maintaining the same in terms of repair, AMC and upgrades. To solve this problem they propose the adoption of Cloud Computing where centrally administered computing capabilities are delivered as services, on an as-needed basis, across the network to a variety of users. They conclude that cloud computing provides an economical, viable, scalable, flexible solution, to the problems of ever growing technology of computing resources. 
Vitkar, (2012) presented a study of a higher education institution who adopted Cloud Computing services to meet the IT needs. This cloud-based IT setup benefited immensely in reducing the expenses related to software licensing and IT staff by $75 \%$. Also, it was noticed that through this approach several other benefits were also achieved that include student's success, institutional performance \& competition and improved research environment leading to innovation. Idowu, (2012) advocates the use of Cloud Computing for sustainable developmental transformations process in higher education, especially in the current worldwide economic meltdown. They foresee that adopting cloud computing will have economic benefits to the institution since budgets cuts can be applied for hardware purchases, licensing software and reduction in the IT staff for administration. The resulting savings from adopting cloud services can be used in other developmental areas of the institution. Also, it will provide an easy access to data and manage the user applications ubiquitously with the help of low end devices like handheld devices and notebooks as opposed to laptops and desktops.

Johnson, et al, (2014) present an evaluation of the knowledge and usage of Cloud Computing in Ghanaian higher educational institutions and also find the users awareness concerning implementation cost, security and privacy issues. To achieve these objectives they conducted a survey and presented their findings. Majority of the survey respondents agreed that the use of cloud technologies will improve the information technology usage in their universities. However, the participants of this research were not certain that the security and privacy of data in the cloud was assured. Most of the respondents also agreed that cloud technology if adopted and implemented will be cost effective and help reduce high IT budgets in higher academic institutions. Masud, (2012) presented a four stage migration roadmap for higher education institutions which have understood the benefits of Cloud Computing and have in principle agreed to switch over from the traditional IT setup to a cloud-based IT model. The first stage involves feasibility analysis, stage two involves benchmarking with other deployments, service provider suitability study is the third stage and migration and maintenance planning is the last stage. They also advice to include a set of essential criteria for cloud-based selection in the roadmap which are: standards functionality, security issues, resource planning, backup and recovery procedures, allocation policies and measurement of cloud services. Masud, et al, (2013) later presented a new architecture of mobile learning system in a Cloud Computing environment enriched with mobile and wireless devices. Since mobile learning systems usually require much hardware and software resources, there are numerous educational institutions that cannot afford such investments, and cloud computing is a viable solution for them.

Kumar, et al, (2013) present a study on educational cloud computing and how the universities and institutions are already taking advantage of it, not only in terms of cost but also efficiency security, reliability and portability. They provide several general examples of cloud computing in education such as Microsoft, Google App, IBM and Amazon. They also present two case studies in the context of Cloud Computing adoption in the Indian education setup, namely, IGNOU (Indira Gandhi national Open University) e-learning project and All India Council for Technical Education (AICTE) partnership with Microsoft Corporation.

\section{Methodology}

A four stage methodology that has to be adopted by HEIs in order to acquire Cloud services and develop a KMS is shown in Figure 1. Stage 1 is to develop an evaluation criteria for choosing a KMS, stage 2 is to develop migration framework for Cloud-based IT setup, stage 3 is to understand the financial, academic and administrative implications and the fourth stage is 
implementation of Cloud-based KMS setup and demonstration of its practical applicability. In the following each stage will be discussed in detail.

The first stage is to design a set of evaluation criteria for choosing a KMS for a HEI. A software like Analytic Hierarchy Process (AHP) can be utilized to evaluate the four major criteria and sub-criteria given in (Greco et al 2013):

a. Knowledge Impact which includes Knowledge Creation, Knowledge Accumulation, Knowledge Transfer and Knowledge Diffusion.

b. Application which includes Personalization, Collaboration and Communication, Integration and Tracking and Monitoring

c. Cost Reduction which includes Capital Expenditures and Operating Expenditures

d. Stakeholder Satisfaction which includes Students, Faculty, Administrators and Service Providers.

In the second stage a Cloud-based migration strategy should be developed, that provides a roadmap consisting of a four step evaluation process as shown Figure. 2.

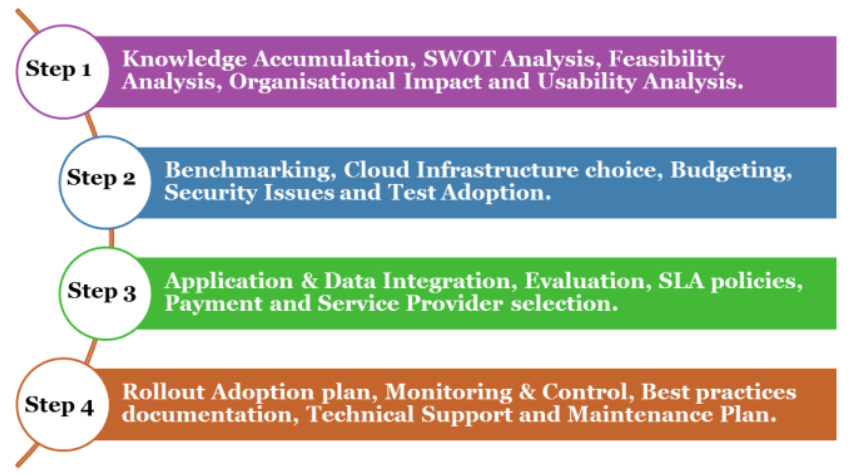

Figure 2: Road map for Cloud-based migration strategy

In Step 1 Knowledge has to be accumulated, SWOT analysis and feasibility analysis should be performed, while addressing the organizational impact and finally its usability analysis should also be performed. In Step 2, a benchmark for similar case must be identified, the cloud infrastructure choice should be decided, a budget should be made for the project, and finally security issues should be addressed before test adoption performed. Step 3 includes application and data integration, evaluation, service level agreement (SLA) policies, payment and service provider selection. Step 4 involves the rollout adoption plan, monitoring and control, best practices documentation, technical support and maintenance plan.

In the third stage a survey will be conducted which will analyze the financial, academic and administrative implications of moving to a Cloud Computing-based IT setup. The steps are shown in Figure. 3. Survey Design should include appropriate questions based on the above stages. Survey conduct includes sending requests for participating in the survey and monitoring the submitted surveys. Survey analysis includes gathering data from the surveys and analyzing the results.

The final stage is implementation and demonstration of the benefits of a Cloud-based KMS and showing its applicability to other higher education institutions. This can be achieved through testbed setup and performing simulation experiments as shown in steps on Figure. 4. 
Traditional Testbed setup includes setting up a server which can support an open source Knowledge Management System (e.g. (openKM)) and then test this system under varying load conditions. Cloud-based setup includes setting up client (low-end machine) which can support a cloud-based simulation software (e.g. (SteelCentral Web Analyzer)) and test this system for web and cloud-based application monitoring. Comparative study involves comparing the performances of the systems in Traditional Testbed setup and Cloud-based setup above, in terms of costs, implementation complexity, stakeholder satisfaction and knowledge management impact.

\section{Impact, Benefits and Potential Risk of Proposed KMS}

Utilization of $\mathrm{CC}$ in general has a positive impact on any organization's performance and undoubtedly include the HEIs as well. The following list provides the outcome of utilizing CC based KMS at HEIs.

- Provides better services to the stakeholders.

- Inculcates organizational change in the HEIs.

- Minimizes operational costs

- Encourages creativity and innovation to mine accurate and crucial information, which may include both tacit and explicit knowledge.

- The IT staff will now be able to focus on enhancing their services to users, whether faculty, students, or administrators rather than losing time in maintenance and repair of IT equipment which will be reduced to the minimum as a result of CC services.

A Cloud Computing based KMS as a tool for HEIs can benefit in many ways and some of them are listed below.

- Enhances process management.

- Can be easily utilized by any educational organization, small or big, public or private, profit oriented or not for profit type which can be diverse in their operations.

- Aid to evaluate alternative ways of developing a new (or upgrade) KMS for a higher education institution.

- Can be utilized as a road map for migrating from a traditional IT setup to a Cloud Computing based system.

Any technological development is associated with potential risks when adapted. However, time and again it has been proved that organizations evaluate the benefits against the potential risks of the technology and acquire it if the benefits surpass the risks. Similarly, CC is also linked with some potentials risk which are generic in nature irrespective of its application area. The prominent among those risks is the risk of data security. Researchers have been developing tools to secure the cloud and are very promising about the future to minimize the risks. 


\section{Case Study and Future Work}

The current overview and methodology presented in this paper is part of an ongoing project at a private university in a Saudi Arabian city, Al-Khobar. Prince Mohammad bin Fahd University (PMU) is a fast growing institution and accommodates more than 5000 students for a variety of undergraduate and few master's programs. Since inception it has maintained its reputation for using the latest technologies to provide excellent services to the students like e-library access, online courseware, Dyned labs, and online registration among others. Currently, the accreditation requirements and the increasing documentation of academic records is posing challenges. Hence a supporting technology is seek out that can streamline all academic processes along with administrative processes. KMS is a viable solution in this scenario that can provide a dashboard like environment for linking all knowledge related activities at any HEI. However, traditional KMS may actually turn into CC based KMS soon to achieve maximum benefits. This development in technology can remain as a solution for a significant amount of time as cloud services are to be utilized by most of the organizations in future.

A proposal is therefore put forward to evaluate the possible benefits of developing a CC based KMS. The proposed methodology has been outlined in this paper that will be furthered as per the schedule. Currently the authors are working on developing a survey to help obtain the critical evaluation criteria (Stage 1) and will soon acquire the necessary data and then proceed to next stage. The final output of this proposed work is to have an evaluation tool that can be utilized as a first step by any small or medium scaled HEI, to decide whether the institute should adapt a CC based KMS or not.

\section{Conclusion}

A four stage model has been proposed in this paper which helps in development of CC based KMS for HEIs. The proposed work applies to the improvement of Knowledge Management in the Higher Educations institutions by application of Cloud Computing IT model. Furthermore, it is aimed at the achieving a sustained development and progress of college and universities in the Kingdom of Saudi Arabia in general and Prince Mohammad Bin Fahd University in particular. The proposed CC based KMS will be a generic tool that can be utilized by any small or medium level HEI to evaluate its fitness to acquire CC services for developing their KMS. 


\section{References}

i. $\quad$ Allee, V., 1997. 12 Principles of Knowledge Management. Training and Development, Volume 51, pp. 11-18.

ii. Analyzer, S. W., n.d. SteelCentral Web Analyzer. [Online] Available at: http://www.riverbed.com/

iii. Bimol, S., Saikia, M. \& Devi, L., 2014. Achieving Knowledge Management through Cloud Computing: A case in higher education, Proceeding of International Conference on Computing for Sustainable Global Development (INDIACom). s.l., s.n.

iv. Brewer, P. \& Brewer, K., 2010. Knowledge Management, Human Resource Management, and Higher Education: A Theoretical Model. Journal of education for Business, Volume 85, pp. 330335 .

v. Coukos-Semmel, E., 2003. Knowledge Management in Research University: The processes and strategies,Proceedings of American Educational Research Association 2003 Annual Meeting. Chicago, s.n.

vi. Cranfield, D. \& Taylor, J., 2008. Knowledge Management and Higher Education: a UK Case Study. The Electronic Journal of Knowledge Management, 6(2), pp. 85-100.

vii. Hassan, N., Hayiyusuh, N. \& Nouri, R., 2011. The Implementation of Knowledge Management System (KMS) for the Support of Humanitarian Assistance/Disaster Relief (HA/DR) in Malaysia. International Journal of Humanities and Social Science, 1(4).

viii. Idowu, S. \& Osofisan, A., 2012. Cloud Computing and Sustainable Development in Higher Education. Journal of Emerging Trends in Computing and Information Sciences, 3(11), pp. 14661471.

ix. Jennifer, R., 2000. Is Higher Education Ready for Knowledge Management?. The International Journal of Educational Management, 14(7), pp. 325-333.

x. Johnson, L. \& Hiran, K., 2014. Cloud Computing in Ghanaian Higher Educational Institutions, Proceeding of International Conference on Science, Technology, Education, Arts, Management and Social Sciences. s.l., s.n.

xi. Kok, A., 2007. Intellectual Capital Management as Part of Knowledge Management Initiatives at Institutions of Higher Learning. The Electronic Journal of Knowledge Management , 5(2), pp. 181192.

xii. Kumar, B., Kommareddy, S. \& Rani, N., 2013. Effective ways Cloud Computing can contribute to Education Success, Advanced Computing. An International Journal, 4(4), pp. 17-32.

xiii. Masud, A., Yong, J. \& Huang, X., 2012. Cloud Computing for Higher Education: A Roadmap, Proceeding of 16th IEEE International Conference on Computer Supported Cooperative Work in Design. s.l., s.n.

xiv. Masud, A., Yong, J. \& Huang, X., 2013. A Cloud Based M-learning Architecture for Higher Education. Archives Des Sciences, 66(1), pp. 755-760.

xv. Milam, J., 2001. Knowledge Management for Higher Education. ERIC Digest, pp. 464-520.

xvi. Open KM, n.d. Open KM. [Online] Available at: http://www.openkm.com/en/

xvii. Petrides, L. \& Nodine, T., 2003. Knowledge Management in Education: Defining the Landscape. s.l.:s.n. 
xviii. Sharma, A. \& Ganpati, A., 2013. Cloud Computing: An Economic Solution to Higher Education. International Journal of Application or Innovation in Engineering \& Management, 2(3), pp. 200206.

xix. Vitkar, S., 2012. Cloud based model for e-Learning in Higher Education, Proceeding of International Symposium on Advanced Engineering Technology. s.l., s.n. 